\title{
Igualdad en la universidad Una tarea sindical
}

María Yamile SocolovskY*

$\mathrm{L}$ as mujeres hemos logrado, tras una larga historia de exclusión de la educación universitaria, una presencia indiscutida en las instituciones académicas. Tanto en el estudiantado como en las plantas docentes, la participación femenina en algunas áreas supera largamente el 50\%. Esta participación, sin embargo, se encuentra fuertemente signada por formas de segmentación horizontal y por la acción notable pero no siempre visible - de condicionantes que determinan la existencia de "techos de cristal", que operan aquí como en prácticamente la totalidad del mundo del trabajo.

Particularmente en América Latina, la situación de las mujeres en el ámbito universitario no ha sido aún suficientemente estudiada, y mucho menos denunciada, incluso por las propias trabajadoras. La desigualdad de género en las universidades es invisibilizada por la cultura competitiva e individualista que acompaña la precarización de las condiciones de trabajo en el ámbito académico. De allí que una acción sindical con perspectiva de género resulte decisiva para identificar una situación que es injusta en sí misma, pero que también resulta un índice claro de la existencia de un conjunto significativo de inequidades y violaciones de derechos para trabajadores y trabajadoras.

La incorporación de los sindicatos de la educación superior a la Red de Trabajadoras de la Educación de la Internacional de la Educación para América Latina ha sido determinante para el comienzo de una tarea en la que las universitarias hemos aprendido mucho de las trabajadoras de otros niveles educativos, cuyas organizaciones, en no pocos casos, tienen una importante experiencia en la lucha por la igualdad de género, y en el desarrollo de estrategias para fortalecer la participación de las mujeres en la vida sindical.

* Profesora y Licenciada - Filosofía. Directora del Instituto de Estudios y Capacitación (IEC), directora de la Federación Nacional de Docentes Universitarios (Conadu) y Secretaria de Formación, Investigación, Proyecto y Estadística de la Central de Trabajadores/ras de la Argentina (CTA). Buenos Aires/ARG. E-mail:<yamilesocolovsky@gmail.com>. 


\section{Las mujeres que trabajan en la universidad}

La presencia de mujeres en las universidades es una realidad. Pero, ¿cuál es la modalidad de nuestra presencia en este espacio laboral? En primer lugar, las mujeres logramos ingresar inicialmente, y somos mayoría, en aquellas áreas ligadas a tareas del cuidado, incluso si la formación académica en ellas apunta en muchos casos a un desarrollo profesional que no implica de manera directa la atención de otras personas. Zonas altamente feminizadas, como la pedagogía y ciencias de la educación, la psicología, o la literatura, coexisten con áreas en las que somos una minoría incómoda, especialmente las ingenierías y otras carreras de orientación técnica. Entre ambas, se despliega una amplia gama de disciplinas en las que la paridad nos coloca sin embargo, nuevamente, en las especialidades consideradas aptas para el rol que la sociedad patriarcal nos asigna, o en aquellas en las que un menor prestigio profesional se vincula con la falta de interés de los varones por desarrollar en ellas sus carreras. Las mujeres médicas son dermatólogas, o nutricionistas; los varones son cirujanos, o cardiólogos. Las mujeres se dedican al derecho de familia, los varones al derecho penal. Por supuesto, estas generalizaciones encuentran, en todos los casos, sus excepciones. Pero se trata precisamente de excepciones a una tendencia que es impulsada por normas no escritas pero efectivas, excepciones que testimonian en trayectorias particulares el alto costo que demanda avanzar en una carrera académica o profesional contra la corriente dominante.

Esta segmentación horizontal se combina, además, con la dificultad de las mujeres universitarias para acceder a los puestos de mayor jerarquía en las universidades; esta limitación es claramente más notoria en aquellas áreas hostiles a la presencia femenina. Las categorías superiores de la docencia y la investigación, jefaturas de cátedra o área, direcciones de laboratorios y equipos de investigación, cargos de autoridad política son mayoritariamente ocupados por varones. Un "techo de cristal" impide a las mujeres progresar en sus carreras académicas y llegar a ocupar posiciones de mayor responsabilidad y reconocimiento, y con mejores remuneraciones. Las categorías que permiten grados más amplios de autonomía profesional, y que, en tanto implican dirigir a otras personas y decidir asignación de recursos y oportunidades, constituyen una fuente de poder político, llevan normalmente nombre de varón.

Una interpretación usual de la "apertura" de ciertas áreas a la presencia femenina atribuye el relajamiento de la exclusión patriarcal a la devaluación de las actividades, títulos, carreras, profesiones. En esta hipótesis, la feminización sucede a la desvalorización social de una actividad; vale decir, llegamos porque nos dejan, porque a los varones ya no les interesa asegurar su exclusividad y dominio allí, porque ya no representa una fuente de prestigio, de poder, de riqueza. También podría, en cambio, sostenerse que esas actividades son desvalorizadas precisamente porque son realizadas por mujeres. Es posible que ambas hipótesis expliquen adecuadamente situaciones diversas, e 
incluso es probable que se encadenen trazando un círculo difícil de romper. Como sea, puede resultar más interesante preguntarse por qué es que las mujeres "eligen" ciertas carreras, ciertas profesiones, y no otras: en este punto, sería importante nuevamente evitar respuestas reductivas: hay mujeres que eligen ingresar en territorios académicos o profesionales que llegan a ser tan hostiles como para que finalmente desistan de sus vocaciones y se retiren a espacios más hospitalarios, incluso si no responden a sus genuinas expectativas, contrariando sus deseos y renunciando a un desarrollo pleno de sus capacidades. En estos casos, lo que suele presentarse como una decisión autónoma de las mujeres (una "auto-exclusión") es en realidad resultado de formas más o menos brutales de expulsión que son injustas y frustrantes para sus víctimas, y que conllevan una enseñanza disciplinadora para todas.

Precisamente por ello, en virtud de esta lección impartida de manera difusa desde la cultura académica, ocurre que muchas mujeres eligen ya condicionadas por esta expectativa, negativa, que nos advierte que el costo de intentar involucrarnos en algunos campos de actividad sería excesivamente alto para nosotras. Otras no llegan siquiera a considerar esta posibilidad, y limitan sus decisiones a un abanico de opciones culturalmente sesgado desde que aprendimos, junto con el lenguaje y las formas más básicas de las relaciones humanas, que estábamos destinadas a cumplir un rol determinado en la sociedad patriarcal. La reproducción de estereotipos de género se encuentra probablemente en la base de la mayor parte de las elecciones que hacemos las mujeres que llegamos a tener la oportunidad de hacer una apuesta a la formación superior.

Estas formas de aparente auto-exclusión - que preferimos considerar como "exclusiones anticipadas", para evitar responsabilizar a las propias mujeres por la imposibilidad o dificultad para afrontar determinadas opciones - es un problema, porque es un comportamiento que oculta el problema anticipadamente, es una estrategia que no perturba los límites culturalmente impuestos. Aquí es decisiva la cuestión educativa desde la primera infancia y a lo largo de toda la trayectoria educativa que la sociedad nos propone antes de llegar a la instancia universitaria. Y esa es una cuestión que las universitarias no hemos elaborado aún de manera suficientemente clara, asumiendo que se trata de una tarea de las maestras y profesoras de la enseñanza básica y media. Las universidades son, sin embargo, en muchos países, responsables de la formación del magisterio y el profesorado. Y aún en los casos en los que no son las universidades los ámbitos directos de formación pedagógica, su influencia en la construcción de los procesos de enseñanza se extiende a todo el sistema a través de la producción de enfoques y contenidos disciplinares. La problemática de género ha ingresado bastante recientemente a las universidades como un objeto de estudio académico, pero permanece aún recluido en ciertas áreas específicas de investigación que se centran especialmente en un examen crítico de la condición de la mujer en la sociedad patriarcal que no está suficientemente atento a lo que ocurre dentro de la propia institución en la que se llevan a cabo esas 
investigaciones. Del mismo modo en que la investigación educativa que se realiza en las universidades se ocupa, con vocación crítica o con pretensión normativa, de la enseñanza en otros niveles del sistema, y no tematiza la misma práctica pedagógica en la propia universidad, estudiamos la cuestión de género como si se tratara de algo que acontece fuera de nuestro propio ámbito de actividad. Ambas limitaciones se suman para incrementar esta ceguera: la falta de una reflexión adecuada sobre la desigualdad de género en la universidad y su influencia en la reproducción del patriarcado, y la ausencia de una crítica sistemática de nuestra propia práctica docente, nos alejan del desafío político-pedagógico de ser parte activa en la construcción de un paradigma educativo emancipatorio, que sustente en una perspectiva integradora de todos los niveles del sistema de enseñanza la contribución de la docencia a la transformación igualitaria de nuestra sociedad.

\section{Las condiciones del trabajo y la desigualdad de género en las universidades}

La cuestión cultural es, sin dudas, determinante de las trayectorias de las mujeres en las universidades, como lo es en general en el mundo del trabajo. Nuestras expectativas ya están, en gran medida, configuradas en un horizonte estrecho que la cultura patriarcal nos propone como un destino. No obstante, las mujeres hemos demostrado sobradamente que no somos pasivamente sujetadas a los roles que una cultura opresiva nos asigna. La resistencia a adecuarnos a este molde encuentra, sin embargo, límites materiales en las condiciones objetivas en las que se desarrolla toda nuestra actividad. En este sentido, es imprescindible prestar atención a las condiciones de trabajo, también en el mundo académico.

La actividad académica se desarrolla en condiciones que suponen para un grupo amplio de trabajadores y trabajadoras de la enseñanza superior niveles importantes de precarización. Esta situación varía entre los distintos sistemas universitarios latinoamericanos, pero muestra semejanzas significativas, que responden a un proceso global resultante de la implementación de reformas de corte neoliberal, que en nuestra región se llevaron adelante especialmente en la década del' 90 pero han seguido desarrollándose, incluso en el marco de procesos políticos de carácter democrático y popular. Esta "inercia" y continuidad de la adecuación de los sistemas universitarios a la lógica del mercado responde fundamentalmente al efecto de una muy difícilmente evitable internacionalización de las actividades académicas, que somete a nuestras trabajadoras y trabajadores al imperio de un conjunto de dispositivos y criterios de validación de su tarea que tienen un carácter notablemente alienante. Para decirlo muy brevemente: la devaluación de la actividad de enseñanza frente a la sobrevaloración de la tarea de investigación, y el sometimiento de ésta a un modelo que, en gran medida, reduce la valoración del 
trabajo académico a la evaluación de resultados contabilizados sobre la base de la publicación de "papers" en revistas internacionales, ha producido una fuerte segmentación en el mundo universitario, entre aquellas personas que se ocupan de tareas de enseñanza en los niveles iniciales (docencia de grado), y quienes se dedican casi exclusivamente a la investigación y sólo se ocupan de la enseñanza en el posgrado. Normalmente, es muy difícil lograr un tránsito "ascendente" entre estos sectores de la actividad, cuyas condiciones son notablemente diferentes. El primer grupo está sometido - de manera creciente - a peores condiciones de trabajo: formas de contratación a término o por horas, sobrecarga de tareas, exceso de horas de aula frente a cursos numerosos, falta de reconocimiento de tiempos rentados para la preparación de clases y recursos de enseñanza, ausencia de oportunidades para el perfeccionamiento docente y disciplinar. Estos trabajadores y trabajadoras, que también hacen esfuerzos para poder incorporarse en el mundo de la investigación, en busca de mayor reconocimiento y de una mejora en la perspectiva de sus carreras, son, además, parias en el sistema político de las universidades, que en general excluye de la participación en el gobierno de las instituciones a quienes trabajan en ellas en estas formas irregulares e inestables. En muchos casos, la falta de una relación laboral adecuadamente formalizada, dificulta también la sindicalización de estos trabajadores y trabajadoras.

La consideración de la problemática de género en las universidades no puede omitir una crítica de estas condiciones estructurales que determinan la forma del trabajo en las instituciones académicas y de la enseñanza superior, porque son las mujeres quienes resultan más perjudicadas por esta situación. Si volvemos a mirar cuáles son las posiciones que ocupan las mujeres en las plantas docentes de las universidades, veremos que ellas están mayoritariamente situadas en la periferia de un sistema cuyo centro - ocupado por una elite académica que disfruta de estabilidad y oportunidades notoriamente mayores para acceder a los recursos materiales y simbólicos que otorgan poder, prestigio y autonomía - es visiblemente masculino. En un ámbito laboral altamente competitivo, las mujeres nos encontramos en clara desventaja para desarrollar carreras que exigen dedicación exclusiva y continuidad. En ausencia de políticas públicas e institucionales que apoyen una distribución más igualitaria de la responsabilidades del cuidado, la mayoría de las mujeres deben afrontar interrupciones en sus carreras que ningún sistema de promoción toma en cuenta como justificadas, y que no son compensadas en ningún proceso de evaluación. Tener hijos o hijas es, para una mujer que trabaja en la universidad, una opción que pone brutalmente en contradicción al trabajo y la familia, y que genera situaciones tremendamente exigentes cuya resolución recae sobre su tiempo, su esfuerzo, y no pocas veces sobre el sacrificio de sus expectativas y deseos.

Por otra parte, esta dinámica refuerza las jerarquías en las relaciones laborales, incrementadas por la acumulación de poder en los pequeños núcleos de conducción, y permite que se multipliquen las situaciones de abuso de autoridad y de acoso laboral. 
En este contexto, la discriminación de género suele pasar desapercibida incluso para las mismas víctimas, que la subsumen en la regla general que rige la modalidad casi feudal en las relaciones académicas. Los casos de acoso sexual son la mayoría de las veces silenciados en este contexto opresivo y fuertemente disciplinador, en el que el predominio de una cultura patriarcal tiende a justificar o negar la violencia que se ejerce en relaciones desiguales que permanecen ocultas bajo la ficción de una igualdad de oportunidades para demostrar la capacidad de ocupar posiciones de mayor jerarquía sobre la única base del "mérito académico". En los últimos años, impulsadas por un contexto en el que avanzaron en algunos de nuestros países las políticas públicas de prevención, denuncia y penalización de la violencia contra las mujeres, algunas universidades han comenzado a desarrollar acciones para institucionalizar cursos de acción ante las situaciones que se producen en estos ámbitos. La creación de áreas específicas para su abordaje, y la producción de protocolos de actuación ante la violencia de género en la universidad constituye un primer e importante paso en el reconocimiento de que efectivamente el problema existe, y representa una oportunidad para fortalecer la perspectiva de género en la acción sindical, que no puede permanecer ajena a esta tarea.

\section{El rol del sindicato y el aporte de la Red de Trabajadoras de la Educación}

De acuerdo con la descripción que hemos propuesto, la discriminación y desigualdad de género en el ámbito laboral de las universidades es una expresión particular de la cultura patriarcal que aún configura las relaciones sociales en general. Sin embargo, las condiciones concretas en las que las universitarias llevan a cabo sus tareas determinan una modalidad específica que es importante identificar para "rescatar del olvido" las injusticias que en este espacio se producen, y poder elaborar estrategias de intervención. El sindicato tiene un rol decisivo en esta tarea, y es imprescindible que se comprenda que la incorporación de la perspectiva de género en la agenda gremial no es una cuestión secundaria ni opcional, sino que debe configurar estructuralmente la totalidad de la definición de la agenda de trabajo de nuestras organizaciones. La defensa y demanda de condiciones dignas de trabajo para todos y todas requiere advertir cuáles son las determinaciones que afectan de manera diferencial a las mujeres, incluyendo la consideración de un conjunto de factores que actúan sobre las oportunidades y condiciones de trabajo desde afuera del ámbito propiamente laboral.

Poder identificar cómo inciden en esta situación las formas de organización del trabajo en la docencia y en la producción de conocimiento, la definición de las modalidades de contratación, de asignación de tiempos de trabajo, de evaluación y promoción en las carreras; poder reconocer la influencia de factores culturales ligados a los estereotipos de género; comprender el peso que tienen las exigencias que ponen en colisión 
trabajo y familia; tomar conciencia y ayudar a sensibilizar sobre la existencia de situaciones de abuso y violencia de género, son algunas de las tareas necesarias para construir una agenda sindical que incorpore demandas igualitarias que requieren el desarrollo de instrumentos de intervención, políticas de apoyo, criterios de compensación, y que pueden constituir además el punto de partida para impulsar procesos aún más profundos de democratización de las relaciones laborales y pedagógicas, y del conjunto de la vida universitaria.

Avanzar en estas definiciones exige un trabajo de esclarecimiento en el interior de las propias organizaciones, en las cuales también persisten importantes desigualdades y prejuicios que dificultan la participación de las mujeres en la vida sindical, y tornan aún más difícil la incorporación de la perspectiva de género. Muchos de los condicionamientos que actúan en el mundo del trabajo se replican en los sindicatos, en los que, pese a la significativa presencia de las mujeres en el sector que representan - mayoritaria en el ámbito educativo, y al menos paritaria en las universidades - son los varones quienes ocupan las posiciones de mayor responsabilidad, visibilidad, y poder. También aquí somos las mujeres las que desempeñamos tareas más exigentes con menor reconocimiento y menor poder de incidencia política. Por eso es fundamental fortalecer la presencia de las compañeras en las organizaciones y politizar su actuación.

La Red de Trabajadoras de la Educación de la Internacional de la Educación para América Latina desarrolla una actividad que ha sido decisiva para lograr avances en este sentido, proporcionándonos, en el intercambio con compañeras de otras organizaciones, y de otros países, una oportunidad excepcional de aprendizaje, reflexión colectiva y acompañamiento, en un esfuerzo que se enriquece en el encuentro de experiencias que nos permiten a todas reconocernos en las preocupaciones, empeños y esperanzas que compartimos. En este ámbito, las mujeres trabajadoras de la educación superior hemos podido aprender mucho de nuestras compañeras maestras, profesoras y auxiliares de docencia de toda América Latina, para comenzar a proponer e impulsar las transformaciones que nos permitan construir la igualdad en nuestros sindicatos y en las universidades, y sumarnos a la larga marcha por la justicia de género en nuestra sociedad. 